\title{
Pancreaticoduodenectomy after Roux-en-Y gastric bypass: A single institution retrospective case series
}

\author{
Ryan Helmick, Ranjodh Singh, Jeffery Welshhans, \\ Elliot J Fegelman, Hassan Shahid
}

\begin{abstract}
Introduction: The unique challenges of pancreaticoduodenectomy in a patient with altered midgut anatomy secondary to Roux-en-Y gastric bypass are seldom discussed in surgical literature. We discuss two such cases at our institution, in light of experiences of other authors. Case Series: The two cases reported here are different from the majority of previously reported cases as remnant gastrectomy was not performed in either patient. However, one patient did receive a gastrostomy tube in the gastric remnant. The essential differences in existing literature are the removal of the gastric remnant in other reports versus a gastrojejunal anastomosis for remnant drainage in our cases, without any clear advantage in either case. Gastric remnant dissection increases operative time in contrast to gastrojejunal anastomosis, which is a nidus for complications postoperatively. With preservation of gastric remnant however, enteral feeding tube can be placed without
\end{abstract}

Ryan Helmick ${ }^{1}$, Ranjodh Singh ${ }^{2}$, Jeffery Welshhans ${ }^{3}$, Elliot J Fegelman ${ }^{3}$, Hassan Shahid ${ }^{4}$

Affiliations: ${ }^{1} \mathrm{MD}$, Resident Physician 5th year, Department of Surgery, The Jewish Hospital, Cincinnati, $\mathrm{OH}$, United States; ${ }^{2} \mathrm{MD}$, Resident Physician 4th year, Department of Surgery, The Jewish Hospital, Cincinnati, OH, United States; ${ }^{3} \mathrm{MD}$, Attending Surgeon, Department of Surgery, The Jewish Hospital, Cincinnati, OH, United States; ${ }^{4}$ Medical Student 3rd year, Department of Surgery, The Jewish Hospital, Cincinnati, $\mathrm{OH}$, United States.

Corresponding Author: Ranjodh Singh, MD, 4777 E. Galbraith Rd, Cincinnati, Ohio, United States, USA 45236; Ph: (513) 686-5466; Fax No: (513) 686-5469; Email: travis287@yahoo.com

Received: 18 June 2012

Accepted: 09 October 2012

Published: 02 July 2013 manipulation of the altered anatomy, as the need arises. Conculsion: Gastric remnant removal is ideal in decreasing the complexity of pancreaticoduodenectomy by reducing the number of required anastomoses. However, there is an increase in operative time required for dissection of the remnant gastric pouch.

Keywords: Gastric bypass, Gastric remnant, Pancreaticoduodenectomy

$$
* * * * * * * * *
$$

Helmick R, Singh R, Welshhans J, Fegelman EJ, Shahid H. Pancreaticoduodenectomy after Roux-en-Y gastric bypass: A single institution retrospective case series. International Journal of Hepatobiliary and Pancreatic Diseases 2013;3:17-21.

Article ID: 100012IJHPDRH2O13

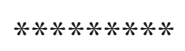

doi:10.5348/ijhpd-2013-12-CS-3

\section{INTRODUCTION}

In the United States due to the growing problem of obesity, many patients are seeking surgical solutions to aid weight loss [1]. Consequently, a growing population of patients has undergone bariatric procedures and has altered foregut anatomy, especially after Roux-en-Y gastric bypass (RYGB). This anatomy poses a diagnostic and therapeutic challenge for foregut and biliopancreatic diseases.

Pancreaticoduodenectomy is a well reported procedure with ample data regarding different techniques and their complication rates. However, pancreaticoduodenectomy after RYGB is rare and only few reports exist in surgical literature. The management 
of the gastric remnant is the challenge when faced with pancreatic resection in this patient population.

The most frequently reported technique of pancreaticoduodenectomy reconstruction involves a remnant gastrectomy. However, some authors use a second Roux limb for drainage of the gastric remnant, while others utilize no drainage procedure. No immediate complications after pancreaticoduodenectomy have been reported to date by these methods. We present our experience to illustrate the fundamental aspects of performing a safe pancreaticoduodenectomy in a patient with prior RYGB.

\section{CASE SERIES}

Two patients with prior RYGB for weight loss presented for pancreaticoduodenectomy. Each was treated by a single surgeon. The patients' records between June 2010 and December 2010 were retrospectively reviewed and analyzed for differences. Patients were assessed first on postoperative morbidity and length of stay, and also compared based on their pathologic findings and surgical techniques. Comparisons were then made between the two patients treated at our hospital as well as the available patient reports in the surgical literature.

Case 1: The first patient was a 71-year-old male with upper abdominal pain for two weeks and history of RYGB for weigh loss in 2006. The RYGB was performed at another institution and thus no follow-up history was available. Initial laboratory examination at the time of admission demonstrated an elevated alkaline phosphatase $215 \mu \mathrm{kat} / \mathrm{L}$, carcinoembryonic antigen CEA $1.7 \mu \mathrm{g} / \mathrm{L}$, and $\mathrm{Ca} 19-954 \mathrm{kU} / \mathrm{L}$. Magnetic resonance cholangiopancreatography (MRCP) demonstrated normal biliopancreatic ductal anatomy and a $6 \mathrm{~mm}$ cystic lesion in the head of the pancreas. The lesion was not evident on contrast enhanced computed tomography (CT) scan. Endoscopic biopsy was not performed in order to avoid potential risk of perforation and increased risk of pancreatitis.

Based on the provisional diagnosis of a cystic pancreatic head mass, we proceeded with a standard pancreaticoduodenectomy involving a distal gastrectomy with removal of the pancreatic head, duodenum, first $15 \mathrm{~cm}$ of the jejunum, common bile duct, and gallbladder (Figure 1). The histopathology showed an intraductal papillary mucinous neoplasm with pancreatic intraepithelial neoplasia grade 2 . The patient was reconstructed in standard fashion with gastrojejunostomy to the remnant stomach. The gastric pouch and roux limb, as well as the jejunojejunostomy, were not altered.

Postoperatively the patient was monitored in the surgical intensive care unit until postoperative day-3. He was quickly advanced to a regular diet. On postoperative day-5, the patient experienced a precipitous decline secondary to bile leak and was returned to intensive care. Computed tomography scan demonstrated a leak from the gastrojejunostomy

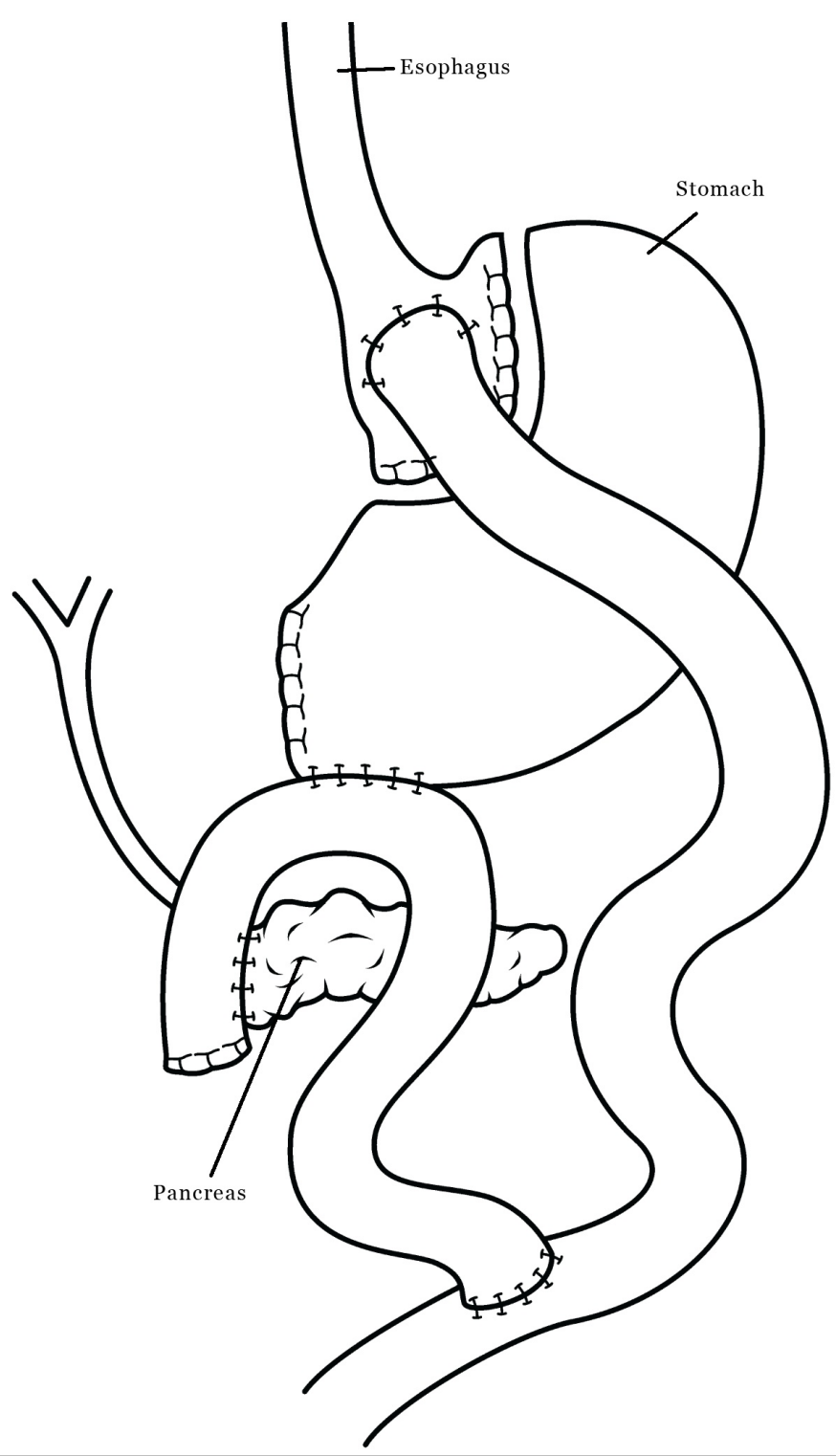

Figure 1: Diagrammatic representation of final reconstruction in pancreaticoduodenectomy after Roux-en-Y gastric bypass.

(Figure 2) and the patient required multiple percutaneous drains, a percutaneous gastrostomy tube, total parenteral nutrition, dialysis and drotrecogin alfa (activated) at $24 \mu \mathrm{g} / \mathrm{kg} / \mathrm{hr}$ for 96 hours. The patient ultimately recovered and was shifted to a skilled nursing facility on postoperative day 53 . This patient is now deceased.

Case 2: The second patient was a 58 -year-old female Jehovah's Witness who presented with a threeday history of epigastric pain with jaundice which started seven days prior to the onset of pain. She also reported elevated liver enzymes assessed by her primary care physician. The patient had undergone a RYGB in 2005 for a BMI of $46 \mathrm{~kg} / \mathrm{m}^{2}$ with a $19 \%$ reduction in BMI at one-year follow-up. A CT scan revealed a $6 \times 14 \mathrm{~mm}$ low attenuation lesion in the head of the pancreas with 


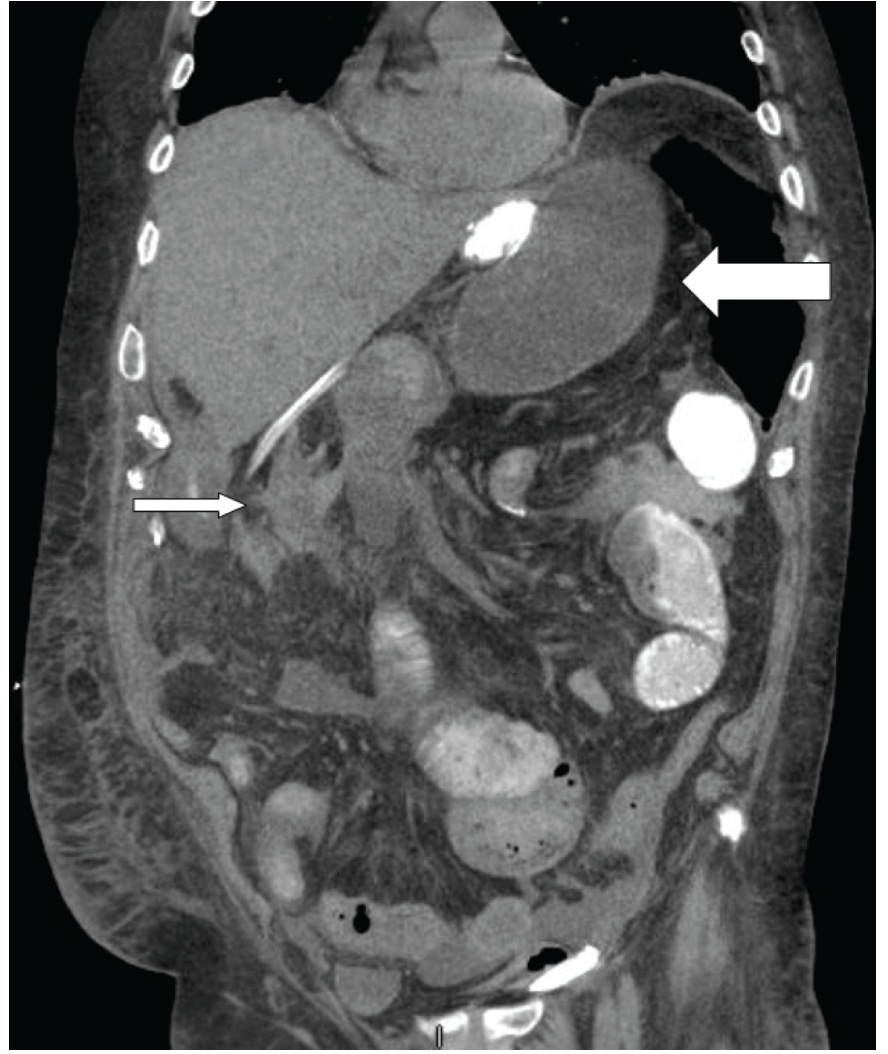

Figure 2: Coronal computed tomography scan of patient on postoperative day-5. The large arrow indicates the distended gastric remnant, while the small arrow indicates the leak from the gastrojejunostomy.

dilated pancreatic and common bile ducts. Magnetic resonance cholangiopancreatography demonstrated a double duct sign but did not show any lesions. She had a percutaneous transhepatic cholangiogram with external drain for biliary decompression. Fluoroscopy via this drain demonstrated a sudden termination of the bile duct. Carcinoembryonic antigent CEA level was 10.0 $\mu \mathrm{g} / \mathrm{L}$ and $\mathrm{Ca} 19-9$ level was <1.0 kU/L. Biopsy performed by interventional radiologist showed atypical cells and inflammatory changes.

There was no metastatic disease and we proceeded with the pancreaticoduodenectomy. Final pathology report showed chronic pancreatitis with fibrosis. The reconstruction was identical to that in Case 1 with the addition of a Stamm gastrostomy tube in the remnant stomach.

Postoperatively the patient was monitored in the intensive care unit with her gastrostomy tube placed to gravity drainage. She was transferred to the ward on postoperative day-2 and was advanced to a regular diet. Her gastrostomy tube continued to gravity drainage until postoperative day- 9 when it was clamped. She was discharged to a skilled nursing facility for physical rehabilitation on postoperative day-10. No complications were reported in the subsequent followups. The most current follow-up, six months after surgery, shows that the patient remains asymptomatic.

\section{DISCUSSION}

The population of morbidly obese patients choosing surgical solutions for weight management is growing by approximately 200,000 patients yearly [1]. This trend has generated a large population with altered foregut anatomy creating diagnostic and therapeutic challenges. The RYGB patients who develop pancreatic head masses present special diagnostic challenges, as the standard pre-operative evaluations with endoscopic ultrasound and endoscopic retrograde cholangiopancreatography are generally unavailable. The techniques to perform endoscopic retrograde cholangiopancreatography or endoscopic ultrasound in the post-bypass patient requires surgical access via the gastric remnant $[2,3]$. Surgically assisted endoscopy is difficult to coordinate and many centers are unfamiliar with the technique. The majority of patients who require decompression of the biliary tree will require percutaneous transhepatic cholangiography with external drainage. Definitive surgical treatment is complicated by the post bypass anatomy and management of the gastric remnant is the key to safe resection.

Rutkoski et al. describe the case of one pancreaticoduodenectomy after bypass for pancreatic adenocarcinoma. The resection was standard and the gastric remnant was drained with a second Roux limb [4]. Although this increased the complexity of the procedure, the remnant was able to drain and the patient experienced no immediate postoperative complications.

The most well reported technique involves resection of the gastric remnant along with the head of the pancreas and duodenum. Nikfarjam et al. and Khithani et al. each reported two patients who underwent pancreaticoduodenectomy with remnant gastrectomy prior to pancreaticobiliary reconstruction $[5,6]$. The largest reported experience is from the Mayo Clinic, where five patients with pancreatic head malignancy underwent pancreaticoduodenectomy. Four of those patients also had remnant gastrectomy, while this was not technically feasible in the fifth patient due to adhesions. None of these patients experienced postoperative complications [7].

Our experience with pancreaticoduodenectomy after RYGB was markedly different between the two patients. One was critically ill with a prolonged hospital stay and significant morbidity, while the other experienced no peri-operative morbidity. The key difference between our patients was placement of a gastrostomy tube at the initial operation. The first patient disrupted his gastrojejunal anastamosis due to stasis in the biliopancreatic limb causing gastric distension. The second patient had a gastrostomy tube present, which functioned as an overpressure valve in the immediate postoperative period. The optimum duration for gastrostomy drainage is approximately seven days assuming drainage is minimal and patient is tolerating the diet. If it is not needed for feeding access, the gastrostomy tube may be removed in two months as is standard for removal of a Stamm gastrostomy. 
A key operative step in patients undergoing pancreaticoduodenectomy after RYGB is decompression of the biliopancreatic limb and specifically the remnant stomach. Other authors have accomplished this via remnant gastrectomy, however our cases would suggest that decompression of the remnant stomach with a gastrostomy will suffice as well. Other reported cases of pancreaticoduodenectomy after RYGB primarily emphasize removal of the gastric remnant, which in effect eliminates the potential for a leak from the gastrojejunostomy. The single case reported by Rutkoski et al. include a drainage procedure for the gastric remnant, the crucial component to this operation [4].

Precautionary measures when operating on such patients are of utmost importance. The indications for surgery should be clearly established. However, these are often difficult in face of limited and altered preoperative workup. Imaging and endoscopic orientation of altered foregut anatomy is important before proceeding with the procedure. A heightened vigilance during postoperative period is warranted to monitor patient for anastomotic leaks.

\section{CONCLUSION}

In patients undergoing pancreaticoduodenectomy after Roux-en-Y-gastric bypass creation of a second roux limb to drain the gastric remnant is not ideal, as the complexity of the operation increases and two anastomoses have the potential for complication. Performing remnant gastrectomy at the time of pancreaticoduodenectomy simplifies the reconstruction, however increases the difficulty of dissection and operative time. Placement of a gastrostomy tube within the gastric remnant achieves the key aspect of safety, namely drainage of the stomach, while limiting the amount of dissection during the resection. This technique preserves the gastric remnant if enteral feeding access is required, without manipulation of the gastric pouch or Roux limb, or placement of a jejunostomy tube.

$* * * * * * * * *$

\section{Author Contributions}

Ryan Helmick - Substantial contributions to conception and design, Acquisition of data, Analysis and interpretation of data, Drafting the article, Revising it critically for important intellectual content, Final approval of the version to be published

Ranjodh Singh - Drafting the article, Revising it critically for important intellectual content, Final approval of the version to be published

Jeffery Welshhans - Drafting the article, Revising it critically for important intellectual content, Final approval of the version to be published

Elliot J Fegelman - Drafting the article, Revising it critically for important intellectual content, Final approval of the version to be published
Hassan Shahid - Drafting the article, Revising it critically for important intellectual content, Final approval of the version to be published

\section{Guarantor}

The corresponding author is the guarantor of submission.

\section{Conflict of Interest}

Authors declare no conflict of interest.

\section{Copyright}

(C) Ryan Helmick et al. 2013; This article is distributed under the terms of Creative Commons Attribution 3.0 License which permits unrestricted use, distribution and reproduction in any means provided the original authors and original publisher are properly credited. (Please see www.ijhpd.com/copyright-policy.php for more information.)

\section{REFERENCES}

1. Trus TL, Pope GD, Finlayson SR. National Trends in Utilization and Outcomes of Bariatric Surgery. Surgical Endoscopy 2005;19(5):616-20.

2. Gutierrez JM, Lederer H, Krook JC, Kinney TP, Freeman ML, Jensen EH. Surgical Gastrostomy for Pancreaticobiliary and Duodenal Access Following Roux en Y Gastric Bypass. J Gastrointest Surg 2009;13(12):2170-5.

3. Lopes TL, Clements RH, Wilcox CM. Laparoscopyassisted ERCP: experience of a high-volume bariatric surgery center (with video). Gastrointestinal Endoscopy 2009;70(6):1254-9.

4. Rutkoski JD, Gagné DJ, Volpe C, Papasavas PK, Hayetian F, Caushaj PF. Pancreaticoduodenectomy for Pancreatic Cancer after Laparoscopic Roux-en-Y Gastric Bypass. Surgery for Obesity and Related Diseases 2008;(4):552-5.

5. Nikfarjam M, Staveley-O'Carroll KF, Kimchi ET, Hardacre JM. Pancreaticoduodenectomy in Patients with a History of Roux-en-Y Gastric Bypass Surgery. Journal of Pancreas 2009;10(2):169-73.

6. Khithani AS, Curtis DE, Galanopoulos C, Jeyarajah DR. Pancreaticoduodenectomy after a Roux-en-Y Gastric Bypass. Obes Surg 2009;19(6):802-5.

7. Swain JM, Adams RB, Farnell MB, Que FG, Sarr MG. Gastric and Pancreatoduodenal Resection for Malignant Lesions after Previous Gastric Bypass Diagnosis and Methods of Reconstruction. Surg Obes Relat Dis 2010;6(6):670-5. 
Access full text article on other devices

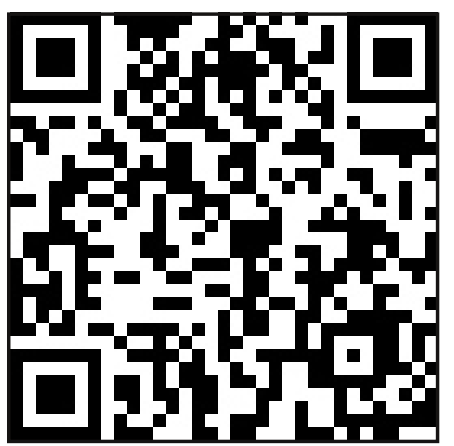

Access PDF of article on other devices

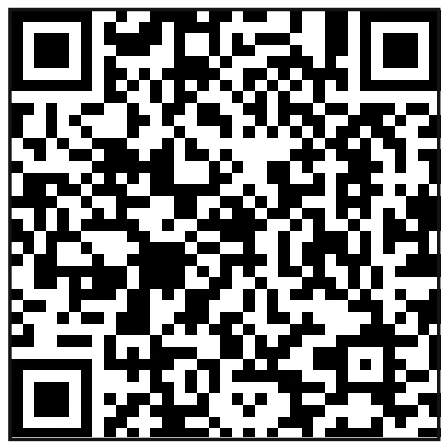

